\title{
Paideusis
}

\section{Education as the Recapitulation of Sense-Making Techniques}

\section{Kieran Egan}

Volume 2, Number 1, 1988

URI: https://id.erudit.org/iderudit/1073418ar

DOI: https://doi.org/10.7202/1073418ar

See table of contents

Publisher(s)

Canadian Philosophy of Education Society

ISSN

0838-4517 (print)

1916-0348 (digital)

Explore this journal

Cite this article

Egan, K. (1988). Education as the Recapitulation of Sense-Making Techniques.

Paideusis, 2(1), 3-13. https://doi.org/10.7202/1073418ar viewed online.

https://apropos.erudit.org/en/users/policy-on-use/ 


\title{
Education as the Recapitulation of Sense-Making Techniques
}

\author{
Kieran Egan, Simon Fraser University
}

Two general ideas have dominated educational thinking during this century. The first and most persistently influential derives from Plato. In this scheme. we must first get clear what we mean by education and what its end will look like. We must then design a curriculum that will carry the child to that end from ignorance and conventional confusions by means of those forms of knowledge that encourage, or entail, the growth of rationality and its power to expose the truth about reality. Each step of the process is determined by our sense of the end - the nature of reality, and of the true, the good, and the beautiful. The curriculum is the causal dynamic that carries the educational process forward. Internalizing the contents of the curriculum, that is, causes the forming of the rational mind.

The second idea was given its most influential expression by Rousseau. He saw it as complementing Plato's. Rousseau argued that the stages of the journey to educated adulthood have distinctive characters of their own that need to be brought to their own perfection or ripeness. Childhood, for example, should not be seen merely as the beginning of a process, a stage defined by ignorance, confusion, and the lack of educated rationality. Childhood has its own form of perfection to whose ripening we must carefully attend. In Rousseau's scheme, the causal dynamic of the educational process is the natural growth or ripening experience of the child to which knowledge must be made to conform if it is to be effectively and usefully learned.

These two ideas have not, however, come easily together. Often enough they have been seen less as complementary insights than as incompatible. Plato's and Rousseau's arguments have so profoundly influenced western thinking about education that they have provided the terms, perhaps also the polarities. of the major educational debates of this century. The trouble is that it is difficult to think of education as a process of potentials ripening according to natural predispositions and at the same time as the accumulation and internalization of knowledge and its logics. It is a bit like the rabbit's or duck's head perception trick. Which is the figure and which the ground? It is as though focusing on the causal role of knowledge or of developing experience inhibits us from perceiving that of the other.

This polarization results, of course, from something of a caricature of Plato's and Rousseau's ideas. It suppresses Plato's sensitivity 
to developmental stages and Rousseau's aim to make a conventional eighteenth century gentleman out of Emile, (and perhaps the less said these days about his aim for Sophie the better). It does, however, bring vividly to the fore a genuine difference of focus and emphasis that has been at the heart of educational conflicts - theoretical and practical - throughout this century. Each of their conceptions of education embodies an important general insight, with neither of which we can sensibly dispense. An aim of educational theory during this century has been to provide a coherent conception of education that takes account of modern conditions and gives due weight to each idea. The need for this has proven easier to identify than to satisfy. however.

The challenge is to construct an educational theory that embodies both the Platonic insight about disciplined knowledge and the Rousseauian insight about natural development. The difficulty lies in showing how they interact. Attempts to overcome this difficulty have involved schemes that simply collapse one to the other, or involve flaccid compromises, or propose principles of interaction that lack the kind of clarity and precision that a useful theory requires. One might collapse one to the other, for example, by arguing that the only kind of "development" of educational significance is that which is generated by accumulating knowledge, or, on the other side, that the kind of knowledge of most importance in education is that which is spontaneously produced in the process of psychological development. Even if one acknowledges the importance of both insights, there remains the problem of locating the causal dynamic of the educational process. Where one locates the dynamic will determine how one characterizes the appropriate interaction between accumulating knowledge and psychological development. Does accumulating knowledge drive psychological development, shape and inform the mind, or does some spontaneous developmental process determine what knowledge can be usefully and meaningfully learned? Or, in terms of the Piagetian/Vygotskian dispute, does learning drive development or development drive learning?

Attempts to bring the two ideas together have been somewhat hindered by the louder part of the debate about education being little concerned with how to overcome the dilemma, or with how to conceive of education so that the dilemma does not arise, but rather more concerned with taking sides on the greater value of one insight over the other. So we have had "traditionalists" vs. "progressivists," "child-centred" vs. "subject-centred," "experience" vs. "basic skills," and so on. One value of these louder voices has been to expose the inadequacy of each insight by itself. On one hand, the very metaphor of education as a journey has been shown to be misleading. Childhood is not something we leave behind. The achievements and experiences of childhood are constituents of our later selves - "the 
child is father to the man," in Wordsworth's compact phrase. "Progressivists" have established the importance of not sacrificing the distinctive qualities of childhood experience to the insistent shaping required to fit children to a given culture, siciety, and economy. Beyond these, we must recognize that we have a nature to whose patterns of development, learning, motivation, and so on, our educational practices must accommodate. On the other hand, "traditionalists" have made clear that nature gives us no particular guidance. We have infinite, indeterminate potentials, and we need to describe precise educalional ends which will provide criteria to guide our choices of which potentials to stimulate and develop. Our fulfilment comes precisely through initiation into a particular culture and fitting into a particular society and its economy: our nature is cultural.

There is a third general idea about education. It is one that has hovered about educational thinking rather indecisively, crystallizing into curriculum proposals only occasionally. This is the idea of education as cultural recapitulation. It had its most influential run in the wake of evolutionary theory during the late nineteenth century, but was quite quickly submerged again under progressivism in North America and the modern education movements in Europe. It has long been obvious that education involves in some fashion the individual's recapitulation of cultural history, but it has not been clear how we could find a basis for describing what might be common to the two processes, nor how we could locate a dynamic that would point up some causal sequence that they share. That is, even though there is a superficial sense in which the individual in being initiated into a particular culture learns what was invented and discovered in the process of that culture's history, it is not clear whether, or in what way, the sequence of the latter process should have an impact on the former.

The bifurcated ramifications of Plato's and Rousseau's insights have encouraged in the study of education somewhat distinct focuses on knowledge accumulation and on psychological development. assuming that separate advances from philosophical and psychological research will somehow be brought together. Clearly recapitulation does not make much sense in terms of knowledge accumulation nor psychological development separately. Even if it were possible, there is no good reason to recommend that the child should recapitulate the accumulation of knowledge as it was invented or discovered in history. How, for example, would one teach geography in such a scheme, and would astrology and alchemy have to precede astronomy and chemistry? Similarly, any theory that sees the individual as recapitulating an historical process of psychological development faces the bizarre requirement of showing Euripedes and Plato as somehow psychological equivalents of modern children. 
Even so, I would like to try to resuscitate cultural recapitulation as a means of coherently encompassing both the Platonic and Rousseauian insights. What is recapitulated, I will suggest, are sensemaking techniques. The sequence of their invention and discovery is connected to individuals' education today by means of the logical and psychological constraints that shaped their historical generation and that shape children's acquisition of them.

To make such a recapitulation scheme even plausible I must try to clarify what l mean by sense-making techniques, and show in what way the historical sequence of the invention and discovery of these techniques was constrained by logic and psychology, and why we should think that the logical and psychological constraints that shape cultural history should shape in a similar way, or cause a similar sequence in, children's acquisition of these techniques.

\section{II}

Crucial to this recapitulation scheme is the nature of sensemaking techniques. We are to focus on these rather than on the more familiar, if still puzzling, phenomena of knowledge and psychological development. What are sense-making techniques? I will give a quick answer to begin with and then return to the question by means of discussing the logical and psychological constraints on their historical generation. Most simply, sense-making techniques are any of the ways we have invented or discovered for making sense of the world or of our experience. They can be small-scale achievements, such as a trick of representation in painting or literature, or linguistic devices such as the subjunctive, or irony, or forms of classification, or more pervasive achievements such as metaphoric fluency, or the story-form, or literacy. The significant feature of sense-making as a category is that it encompasses both logical and psychological dimensions. Acquiring sense-making techniques does not distinguish accumulating knowledge from psychological development. Making sense of something implies both an accumulation of knowledge and a psychological development.

Trying to coalesce the logical and psychological, knowledge and development. may seem a peculiar move when so much of our energy in educational discourse has gone precisely into distinguishing these, in order to distinguish also conceptual from empirical concerns and so bring to bear appropriate methods 10 deal with each of them separately. The distinction between the conceptual and the empirical has been fundamental to assumptions of how best to go about studying education, and it is one that is reified even in the organizational structure of most departments and faculties of education. Implicit in this recapitulation scheme, then, is the assumption that the kinds of distinct philosophical and psychological questions currently prominent 
in education discourse, while philosophically and psychologically most interesting. are not questions for whose answers we should wait in order to get on with our educational business. Indeed the trouble with education as an area for the application of philosophical and psychological methods of research is that empirical and conceptual issues are knotted up in ways that make it difficult, perhaps impossible, to get anywhere by addressing one or the other separately. And, as with the Platonic and Rousseauian insights, and for much the same reasons, the products of these two areas of research in education have had difficulty coming together.

The apparent oddity of trying to coalesce considerations of knowledge and of mind might be mitigated a little further by reflecting on how difficult that distinction is to sustain in practice anyway. While it is much exercised as a kind of demarcation line between philosophers' and psychologists' areas of expertise, we might recall the problems involved in the various attempts to characterize minds and knowledge separately. We know minds almost entirely from what they do with knowledge - the mind is like a transparent organism whose structures only become visible when it ingests the dye of knowledge, but it has proven immensely difficult to establish whether whatever structures we can then make out are a property of the mind or of the knowledge. Similarly we need to remember that we store encoded data, not knowledge, in books and computers. The only proper home of knowledge is a human mind and its forms are tied up with emotions, imagination, intentions, and all kinds of things that make it much less accessible to precise analysis than is encoded data. So the attempt to coalesce in the category of "sensemaking" what are usually distinct focuses on minds or knowledge, on empirical or conceptual questions, on logical or psychological concerns, is not necessarily to be seen as a perverse straining to overcome some invariably fruitful distinction. It is an attempt to ignore a distinction that is enormously difficult to make in practice and one whose value to education is nothing like as clear as its prominence would suggest. Focusing on sense-making, then, leads us away from trying, on the one hand, to characterize some "natural psychological reality, in terms of which we must understand the development of knowledge," as the most Rousseauian of psychologists puts it ${ }^{1}$. and away from. on the other hand, trying to describe forms of knowledge and the prerequisite structures that determine the sequence in which it can be learned. The focus on sense-making does not lead us to suppress logical and psychological concerns, however, rather it leads us to suppress the common distinctions made between them. Instead, it focuses us on the interlacement of knowledge accumulation, psychological development, and cultural history.

What is meant by logical and psychological constraints of the historical development of sense-making techniques? The U.S. space 
shuttle was possible only after the invention of the vacuum flask, the machine gun similarly required the clock, the representational style of leonardo required the technical developments of Giotto, the historiography of Thucydides required the forms articulated by Herodotus, Boolean algebra required Pythagorean harmonies, and so on. The causal sequences in these processes are very complex and very difficult for us to pin down, but the sequences are not arbitrary or accidental; 'Thucydides' history could not have preceded Herodotus', nor could Leonardo's style have preceded Giotto's, nor the space shuttle the vacuum llask, and so on. In writing histories of technology, or painting, or various forms of inquiry, we try to characterize particular achievements and try to expose necessary prerequisites of later developments, thereby exposing a part of the causal dynamic of the changing forms. But such prerequisite structures are never enough by themselves to account for any particular achievement; we need to take into account psychological, social, and other considerations. In mathematics. for example, we have tended to rely heavily on a sense of an unfolding logic in the subject, (which individuals have discovered, not invented). In accounting for technological developments, we find a logic in the sequence of inventions, but one whose shape requires descriptions of social circumstances and occasionally the psychological quirks of inventors. In a case like historiography the logical causal process is somewhat opaque and we focus more on the social, political. ideological, and psychological conditions of the writer. In the arts, the logical element in the reshaping of inherited forms tends to take a subsidiary place to psychological concerns, articulated perhaps in terms of the imaginative life of the artist.

There remains considerable opaqueness concerning causality in virtually all areas of cultural history. In part this is due to the complex ways in which logical and psychological factors both play roles and to our inability to neatly separate out the logical from the psychological. In the historical development of historiography, for example, we can try to specify particular logical developments; Thucydides provides a more "scientific" kind of explanation of events than is to be found in Herodotus' more story-like narrative, and it is a kind of explanation explicitly elaborated due to Thucydides' sense of the inadequacies of Herodotus'. But we cannot describe some precise logical entailments here, even while recognizing a kind of logical accumulation. Accounts of the changes between the two forms of historiography will need also to consider social and psychological factors, and these will be knotted up with the logical in complex ways. We may try to describe, for example, a kind of psychological predisposition also at work determining the development of historiographical forms. The Herodotean account is one in which brave little Athens, representing Liberty and various other virtues, fought against and defeated the brutal despotic Persian empire. The roman- 
tic, audience-gratifying form of Herodotus' history is psychologically less sophisticated than Thucydides' austere attempt to discover the truth about the historical process in general. We are, it can be argued, psychologically predisposed to use the past first for selfglorification and only later develop less egocentric uses for history. 2

This confusion of the logical and psychological as causal factors in cultural history does not, however, make the task of a cultural recapitulation scheme equivalently difficult. Despite the opaqueness concerning causes, what remains relatively clear is the effects. The sequence of the invention and discovery of sense-making techniques was constrained and shaped by logic - certain inventions or discoveries required certain knowledge to be in place to make them possible, and constrained and shaped by human psychology - certain directions are followed rather than others because of the nature of our hopes, fears, intentions, and so on. For our educational purposes, we do not need to factor out the psychological from the logical components in the development of sense-making techniques. It is enough to be able to describe their effects in the sequence of the development of those techniques.

Even if we could provide a description of the historical sequence in which sense-making techniques were developed, why should such a description have any relevance to education? It would be relevant because the individual's ability to acquire sense-making techniques is constrained and shaped by exactly the same forces that have constrained and shaped their historical generation. If $\mathrm{it}$ is the case that certain sense-making techniques could not be conceived before others were in place, then this condition holds on the individual no less than in history; the logic of subjects does not let up, nor does human psychology. If a Thucydidean form of historical understanding is in some complex way a product of an Herodotean form, then in our education we will have to recapitulate the Herodotean form prior to achieving the Thucydidean. Making sense of history in a Thucydidean way both logically and psychologically requires the prior development of the Herodotean way.

But why can we not in our education begin with the more sophisticated form and ignore the discarded prior stages? We are constrained to recapitulate the historical sequence because the prior stages are not discarded. They remain as constituents of the more sophisticated forms. The Thucydidean form of historical understanding does not simply replace or displace the Herodotean; it absorbs significant parts of it, and the latter is prerequisite to the former.

So I am identifying "sense-making" as a centrally appropriate category for educational inquiry, and 1 am identifying. as largely definitive of cultures, the set of techniques they have developed for making sense of the world and of their experience. To borrow some useful jargon, the set of sense-making techniques that constitute our 
culture exists in the present in a complex synchronicity. They were generated diachronically. Education is the process in which the individual acquires these techniques, or a sub-set of them, diachronically. As they are acquired they coalesce in the individual to an analogous synchronicity as they exist in the culture. The dynamic that has determined the diachronic sequence of their invention and discovery in our culture is constrained and shaped by the interaction of logical and human psychology. The sequence in which knowledge ran be elaborated in human minds is constrained by the logic of the various forms of knowledge acting together with the predispositions of human psychology. These same forces whose action we see in cultural development shape and constrain the individual's acquisition of the sense-making techniques available in the culture. In becoming educated, then, we are constrained to recapitulate the sequence whereby in our cultural history these sense-making techniques were invented and discovered. Now, we do not need to recapitulate this sequence if we are not to acquire the fullest range of these techniques - if, that is, we are not to become educated. But if we are to become educated, if we want to maximize our acquisition of the range of sense-making techniques available to us in our culture, then we need to recapitulate the sequence of their historical development. To fail to acquire an Herodotean form of historical understanding, for example, is to fail to acquire prerequisite constituents of a. Thucydidean form, which in turn involves prerequisite constituents of a modern sophisticated historical understanding.

III

If cultural history is going to provide guidance to the curriculum, does this not mean that astrology must precede astronomy. alchemy precede chemistry, magic precede physics and medicine, and myth precede philosophy and literature? Such a conclusion might be encouraged by nineteenth century recapitulation schemes, and might be encouraged by our habitual focus on knowledge accumulation and psychological development separately. Let us consider, for example, whether we are reduced by a cultural recapitulation scheme to the absurdity of requiring astrology to precede astronomy. We would be required to follow such an odd curriculum if it was the particular knowledge content of our cultural history that we are going to recapitulate. But in this scheme, our focus is on sense-making techniques. What we see in the move from astrology to astronomy is indeed an accumulation of knowledge and, crucially, theory replacing story. If, however, we consider astrology in terms of sense-making, we will focus on the cultural achievements embedded in astrology which contribute to enhancing our ability make sense of the world. We tend to dismiss astrology as nonsense, as the irrational predeces- 
sor whose displacement made astronomy possible. But, again, the move from astrology to astronomy cannot be understood if seen simply as a displacement; astronomy grew out of astrology. Our focus is on what survived in that transition. The cultural achievement of astrology was, to put it generally, the imaginative search for meaning in the stars, the consequent observation of order and pattern in complex phenomena, and the attempt to report that order in a memorable and personally meaningful form. It is that we will try to recapitulate in providing an engaging and meaningful access to astronomy. Identifying the cultural achievement that preceded and gave early shape to astronomy enables us to formulate a principle of the construction of an early curriculum in astronomy. Evoking, stimulating, and developing the imaginative search for meaning in the stars does not require that we begin with astrological stories from Greek, Norse, or other mythologies - though we would be a bit obtuse to overlook their possible educational uses in encouraging an initial engagement with astronomy. But we might equally well begin with the most recent findings about the Big Bang, quasars, pulsars, black holes, and so on - if presented in the appropriate form.

What this scheme provides for determining the curriculum, then, is not historically sequenced bodies of knowledge, but rather historically sequenced layers of sense-making techniques. The historical sequence is determined not because of its historicity but because it embodies and reflects the logical and psychological constraints on the development of sense-making techniques. Astrology shows us sensemaking techniques that are prerequisite to and constituent of astronomy. However sophisticated an individual's understanding of astronomy, the imaginative search for meaning, the observation of pattern and order, and so on, will remain fundamental constituents of that understanding.

But astrological stories were replaced by astronomical theories, and if we are to recapitulate this cultural enhancement of our sensemaking capacities then stories must surely give way to theories? And as one seems incompatible with the other, surely this involves straightforward displacement? If our initial exploration of the stars is to be dominated by our storying capacities, and these are somehow to persist throughout our education in astronomy, then surely the development of theory will be prevented? Stories cannot "coalesce" into theories. Is this not a fundamental objection to a scheme of recapitulating an historical sequence of sense-making capacities? I think not. The story form can be used to make a particular kind of sense of any phenomena - a kind of sense that involves affectively orienting us to the story's contents. The development of theory involves attempts at a more precise, literal. objective grasp on phenomena. The kind of sense that theories try to make takes place, as it were, within contexts shaped by our storying techniques. An 
imaginative search for meaning in the cosmos need not interfere with our development of increasingly sophisticated theories about black holes. Indeed, we constantly co-ordinate the two kinds of sensemaking. Our storying capacities do not go away with the development of theories: they provide the contexts of meaning in which theories make more precise sense of their limited phenomena of interest; they provide the ties between theoretically informed knowledge and our lives.

IV

The conception of the aim of education implicit in a cultural recapitulation scheme of this kind does not derive from identifying the characteristics of those conventionally considered best educated, nor on inferences from studies of the nature and forms of knowledge or from theories of psychological development, but is rather arrived at by identifying the major achievements in our cultural history that have extended our capacity to make sense of the world and of experience. These major achievements, again, are not be characterized in terms of changing mental structures, nor in terms of the contents of the particular achievements themselves, but, rather, to fuse these into a way of characterizing sense-making techniques in a form that is equally adequate for discussing cultural history and individual development. 3

The area of study that this scheme finds most useful for education, then, is cultural history. It follows that rather less should be expected from the current dominant research traditions concerned with knowledge and psychological processes than their considerable scale seems to promise. Education seems unlikely, from this perspective, to be improved by some new findings about children or learning, development, motivation, or about the nature of knowledge. Education seems likely to benefit more, to echo Wittgenstein, not by getting new information but by rearranging what we have known all along.

Education in these scheme, then, is the sequential accumulation of the sense-making techniques available in our culture. This is a recapitulationary scheme because it embodies an argument that the sequence in which these techniques can be accumulated by the individual is reflected from the sequence in which they were generated in our cultural history. The tie between the two - cultural history and individual development - is located in the logical and psychological constraints which have shaped the historical generation of these techniques and which also shape the sequence in which the individual can accumulate them. 


\section{Notes}

${ }^{1}$ Richard E. Ripple and Verne N. Rockcastle (Eds.), Piaget rediscovered. (Ithaca, New York: School of Education, Cornell University, 1964), p. 9.

${ }^{2}$ C.R. Hallpike, The foundations of primitive thought (Oxford: Clarendon, 1979).

${ }^{3}$ Kieran Egan, Individual development and the curriculum (London: Hutchinson, 1986) and Primary understanding (London and New York: Routledge and Kegan Paul. 1987). 